\title{
Giant Magnetic Quantum Oscillations in the Thermal Conductivity of TaAs: Indications of Chiral Zero Sound
}

Junsen Xiang, ${ }^{1,2}$ Sile Hu, ${ }^{1,3}$ Zhida Song, ${ }^{4,1}$ Meng Lv, ${ }^{1,3}$ Jiahao Zhang, ${ }^{1,3}$ Lingxiao Zhao, ${ }^{1,3}$ Wei Li, ${ }^{2}$ Ziyu Chen, ${ }^{2}$ Shuai Zhang, ${ }^{1}$ Jian-Tao Wang, ${ }^{1,3}$ Yi-feng Yang, ${ }^{1,3}$ Xi Dai, ${ }^{5}$ Frank Steglich, ${ }^{1,6}$ Genfu Chen, ${ }^{1,3,7}$ and Peijie Sun $\oplus^{1,3,7, *}$
Beijing National Laboratory for Condensed Matter Physics, Institute of Physics,
Chinese Academy of Sciences, Beijing 100190, China
${ }^{2}$ Department of Physics, Key Laboratory of Micro-Nano Measurement-Manipulation and Physics,
Beihang University, Beijing 100191, China
${ }^{3}$ University of Chinese Academy of Sciences, Beijing 100049, China
${ }^{4}$ Department of Physics, Princeton University, Princeton, New Jersey 08544, USA
Department of Physics, Hong Kong University of Science of Technology,
Clear Water Bay, Kowloon, Hong Kong
${ }^{6}$ Max Planck Institute for Chemical Physics of Solids, O1187 Dresden, Germany
${ }^{7}$ Songshan Lake Materials Laboratory, Dongguan, Guangdong 523808, China

(Received 9 February 2019; revised manuscript received 16 July 2019; published 28 August 2019)

Charge transport of topological semimetals is the focus of intensive investigations because of their nontrivial band topology. Heat transport of these materials, on the other hand, is largely unexplored and remains elusive. Here, we report on an observation of unprecedented, giant magnetic quantum oscillations of thermal conductivity in the prototypical Weyl semimetal TaAs. The oscillations are antiphase with the quantum oscillating electronic density of states of a Weyl pocket, and their amplitudes amount to 2 orders of magnitude of the estimation based on the Wiedemann-Franz law. Our analyses show that all the conventional heat-transport mechanisms through diffusions of propagating electrons, phonons, and electron-hole bipolar excitations are far inadequate to account for these phenomena. Taking further experimental facts that the parallel field configuration favors much-higher magnetothermal conductivity, we propose that the newly proposed chiral zero sound provides a reasonable explanation to these exotic phenomena. More work focusing on other topological semimetals along the same line is badly called for.

DOI: 10.1103/PhysRevX.9.031036

\section{INTRODUCTION}

Charge and heat conduction define two entangled fundamental transport properties of a conducting solid. Controllable manipulations of both are essential for various functionalities of pertinent materials. For the recently discovered topological semimetals, electrical transport has revealed distinct topologically nontrivial properties, like the chiral anomaly in the longitudinal magnetoresistance (MR) [1-4]. On the other hand, thermal transport, in particular, thermal conductivity, remains largely unexplored, mainly due to the technical difficulties of precise thermal management at low temperatures. In addition, such work has been so far considered uninteresting, because the

\footnotetext{
* Corresponding author. pjsun@iphy.ac.cn

Published by the American Physical Society under the terms of the Creative Commons Attribution 4.0 International license. Further distribution of this work must maintain attribution to the author(s) and the published article's title, journal citation, and DOI.
}

Subject Areas: Condensed Matter Physics, Topological Insulators thermal conductivity may be estimated from the electrical conductivity through the Wiedemann-Franz (WF) law.

Indeed, for most electrical conductors, the WF law can provide a straightforward and reliable approach to the electronic contribution $\kappa_{e}$ of the thermal conductivity based on the electrical conductivity $\sigma$ :

$$
\kappa_{e} / T=\sigma L_{0},
$$

with the Sommerfeld value of Lorenz number $L_{0} \equiv\left(\pi^{2} /\right.$ 3) $\left(k_{B} / 2\right)^{2}=2.44 \times 10^{-8} \mathrm{~W} \Omega \mathrm{K}^{-2}$. The WF law holds in the case of dominant elastic scattering, e.g., from static defects as realized at sufficiently low temperatures [5]. Within the past few years, it was also found that the charge and heat conduction of the relativistic Dirac or Weyl fermions may deviate from the WF law [6-11]. For example, it was shown very recently that hydrodynamic transport by relativistic Weyl fermions in a Weyl semimetal $\mathrm{WP}_{2}$ can lead to $L / L_{0}<1$ in the temperature range $T<200 \mathrm{~K}$ [10]. On the other hand, for the electron-hole plasma at the charge-neutrality point in graphene, 
considered to form a strongly coupled Dirac fluid, hydrodynamic transport is found between $T=50$ and $80 \mathrm{~K}$ to result in a much enhanced Lorenz number $L \approx 22 L_{0}$ [11].

Large magnetic quantum oscillations (MQOs) in thermal transport properties, like the Seebeck and Nernst effect, have recently been detected and found to complement the conventional techniques like the Shubnikov-de Haas $(\mathrm{SdH})$ effect of $\sigma(B)$ [12-15]. In contrast, the thermal conductivity $\kappa(B)$ has only rarely been used in this respect. An estimate of the MQOs in $\kappa_{e, \mathrm{WF}}(B)$, the electronic thermal conductivity derived from $\sigma(B)$ utilizing the WF ratio $L_{0}$, is generally too small to be detected. The very few examples of $\kappa(B)$ MQOs hitherto reported include a bismuth single crystal [16] and GaAs/AlGaAs heterostructures containing a two-dimensional electron system (2DES) [17]. Here, the amplitude of the MQOs compared to the respective zerofield thermal conductivity $\kappa_{0}$ is less than $8 \%$. However, for the Weyl semimetal $\mathrm{NbP}$ [18], where $\kappa(B)$ MQOs are also observed, it is found to amount to about $30 \%$ of $\kappa_{0}$. A conventional electronic contribution in accord with the WF law, a modulated lattice thermal conductance through electron-phonon $(e-p)$ interaction in a quantum oscillating 2DES, or a large electronic bipolar-diffusion conduction, respectively, are discussed as possible origins of this remarkable observation.

In this work, we report the experimental observation of unprecedentedly large MQOs in the magnetothermal conductivity $\kappa(B)$ of TaAs, a prototypical, recently identified Weyl semimetal [19-22]. The $\kappa(B)$ MQOs exhibit a frequency $(F \approx 7 \mathrm{~T}$ for $B \| c$ ) which is characteristic for a Weyl fermion pocket that is already confirmed in the bulk state of TaAs [19,22]. The amplitude of these MQOs turns out to be surprisingly large; it amounts to more than $300 \%$ of the value of $\kappa_{0}$ when the magnetic field is aligned along the thermal current, i.e., $B\|d T\| c$. Moreover, these oscillations are antiphase to those observed in the electrical conductivity $\sigma(B)$. Our detailed analysis indicates that such giant $\kappa(B)$ MQOs are incompatible with any wellestablished heat conduction mechanism in solids, such as the diffusion contributions by phonons and conduction electrons as well as the contribution by bipolar, electronhole symmetric excitations near a charge-neutrality condition. Here, we show that the main features of the observed $\kappa(B)$ MQOs in TaAs can be ascribed to the recently proposed neutral bosonic excitation, i.e., field-induced chiral zero sound (CZS) associated with the exotic chiral magnetic effect in Weyl semimetals [23].

\section{MAGNETOTHERMAL CONDUCTIVITY}

We employ two TaAs samples cut from the same single crystal grown by chemical vapor transport [19]. They are rectangular shaped, with the long edge either along the $c$ axis [denoted as TAc, dimension $0.3 \times 0.8 \times$ $2.2 \mathrm{~mm}^{3}$; cf. Fig. 1(a)] or along the $a$ axis (TAa, $\left.0.4 \times 1.0 \times 2.1 \mathrm{~mm}^{3}\right)$. Thanks to the large sample size that
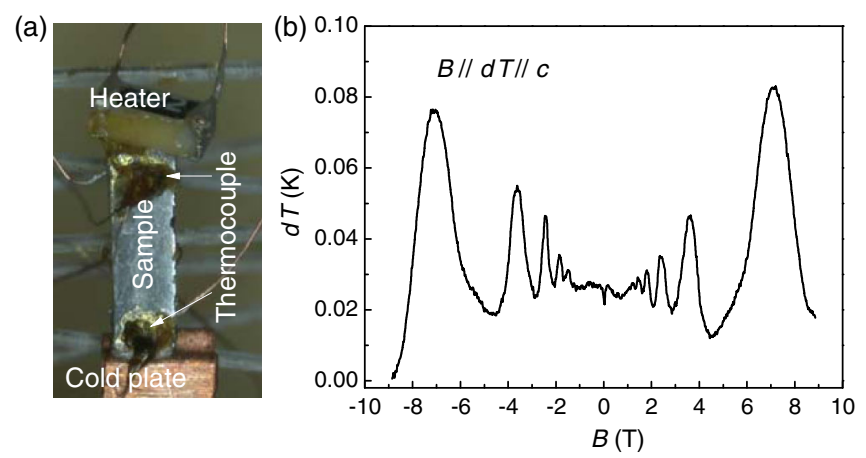

FIG. 1. Thermal conductivity measurement configuration and raw experimental data of temperature gradient $d T(B)$. (a) TaAs sample (TAc) mounted onto a copper cold plate. A chip resistor of $2000 \Omega$ is used as a heater and a thin $(\phi=25 \mu \mathrm{m})$ chromel $\mathrm{AuFe}_{0.07 \%}$ thermocouple for detecting $d T$. Manganin wires $(\phi=25 \mu \mathrm{m})$ used for simultaneous electrical resistivity measurements are connected to the back side of the sample by spot welding. See Sec. VI for more details on the thermal transport measurements. (b) Raw experimental values of $d T$ recorded in a continuous field scan between $B=-9$ and $9 \mathrm{~T}$ at $T=2 \mathrm{~K}$, with a fixed heating power. Both the heat current and magnetic field are applied along the $c$ axis.

allows for configuring multiple thermal or electrical contacts and in order to reduce analytical errors, simultaneous measurements of electrical resistivity $\rho$ and Seebeck coefficient $S$ are carried out in addition to $\kappa$. Upon scanning the magnetic field, the temperature difference $d T(B)$ across the sample oscillates significantly; see Fig. 1(b). In view of the constant heating power $Q$ at the high- $T$ end of the sample, this oscillation signifies pronounced quantum oscillations of $\kappa(B)$; see Fig. 2(b). The latter are crosschecked by employing two types of temperature sensors, i.e., a field-calibrated thermocouple and a pair of Cernox thermometers. The results obtained this way are in very good agreement; see Fig. S2 in Supplemental Material [24]. As shown in Fig. 1(b), the signal of $d T(B)$ is nearly symmetric for positive and negative fields, hinting at good thermal stability during the whole measurement process. In order to eliminate the weak field-odd, thermal Hall components, we take the mean value of $d T(B)$ measured for positive and negative fields throughout this paper. The value of $\kappa$ measured at $B=0$ fits well to our previous results [25].

In Fig. 2, we compare the isothermal electrical and thermal conductivities $\sigma_{\|}(B)$ (a) and $\kappa_{\|}(B)$ (b) of sample TAc. The subscript $\|$ denotes a parallel alignment of the electrical or thermal current and the magnetic field. Note that in a parallel field configuration there is no Hall conductivity, and $\sigma_{\|}(B)=1 / \rho_{\|}(B)$. Here, $\sigma_{\|}(B)$ rather than $\rho_{\|}(B)$ is employed in order to facilitate the following discussions. The strong decrease of $\sigma_{\|}(B)$ at low fields $(\mathrm{MR}>0)$ changes to a weak increase $(\mathrm{MR}<0)$ at $B>1 \mathrm{~T}$, characteristic of the chiral anomaly arising from charge pumping between Weyl nodes of different chirality 


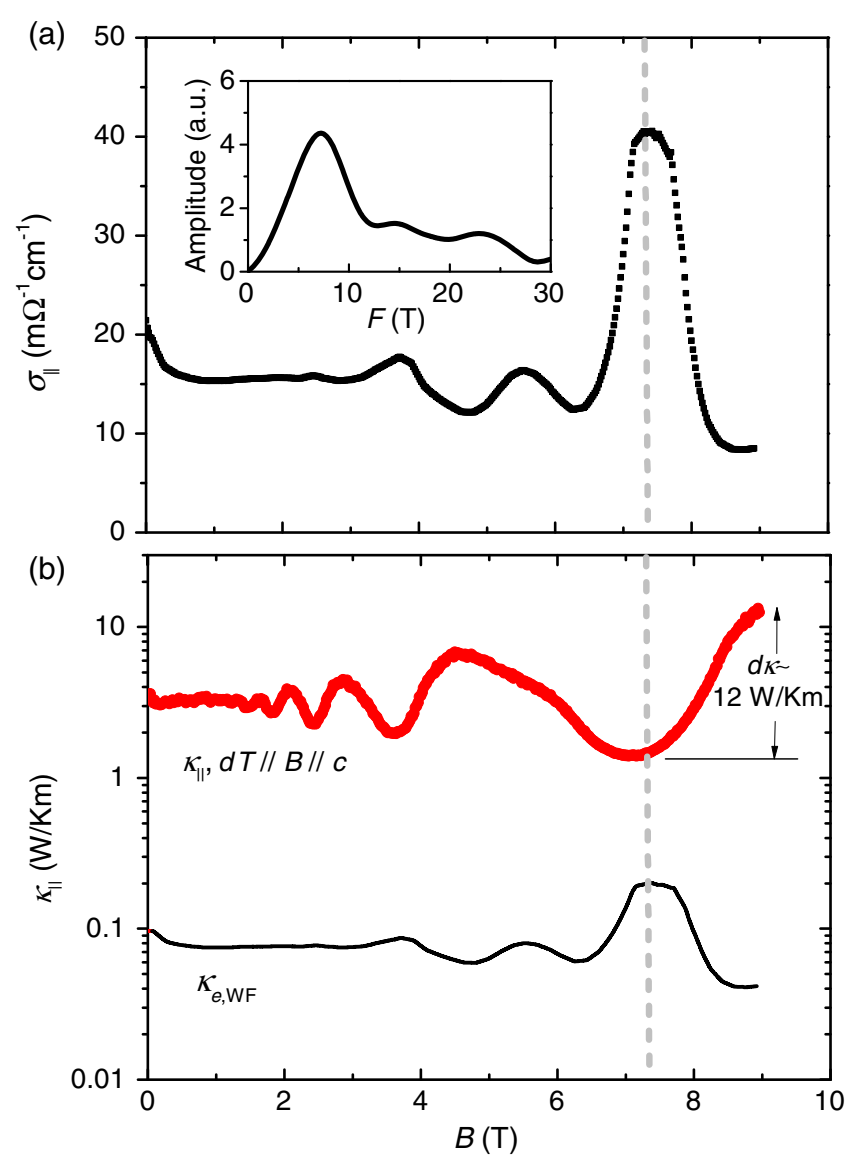

FIG. 2. Comparison of quantum oscillations in $\sigma_{\|}(B)$ (a) and $\kappa_{\|}(B)$ (b) measured at $2 \mathrm{~K}$ for sample TAc. In a parallel field configuration, $\sigma_{\|}(B)=1 / \rho_{\|}(B)$ due to the absence of Hall conductivity. The $\mathrm{SdH}$ oscillations in $\sigma_{\|}(B)$ reveal three characteristic oscillation frequencies (see FFT analysis shown in the inset), in full agreement with literature data [19,21,22]. The MQOs of $\kappa_{\|}(B)$ (b) measured in the parallel field configuration have one dominating frequency of $F \approx 7 \mathrm{~T}$. Also shown in (b) is the calculated $\kappa_{e, \mathrm{WF}}(B)$ from $\sigma_{\|}(B)$ using the WF law. The oscillation amplitude of $\kappa_{e, \mathrm{WF}}(B)$ is 2 orders of magnitude smaller than that of the measured ones [red points in (b)]. Note the logarithmic vertical axis in (b). The dashed line marks the position of the last extremum, where the measured $\kappa_{\|}(B)$ exhibits a minimum, whereas $\sigma_{\|}(B)$ and the calculated $\kappa_{e, \mathrm{WF}}(B)$ assume a maximum, with opposite phases (phase difference $\pi$ ).

[19]. Significant MQOs of $\sigma_{\|}(B)$ at higher fields $B>2 \mathrm{~T}$ reveal several characteristic frequencies, i.e., $F \approx 7,15$, and $23 \mathrm{~T}$, that are consistent with current knowledge on this material [19-22]; cf. the inset in Fig. 2 for the fast Fourier transform (FFT) spectrum. The dominating frequency $(F=7 \mathrm{~T})$ is due to a tiny hole-type Fermi pocket enclosing a Weyl node [19]. Both the magnetization $M(B)$ and the simultaneously measured $S_{\|}(B)$ show marked MQOs as well, with the same dominating frequency as derived from electrical conductivity; see Figs. S3 and S4 [24].

As shown in Fig. 2, the MQOs in $\kappa_{\|}(B)$ are substantially more pronounced, especially at $B<4 \mathrm{~T}$, than those in
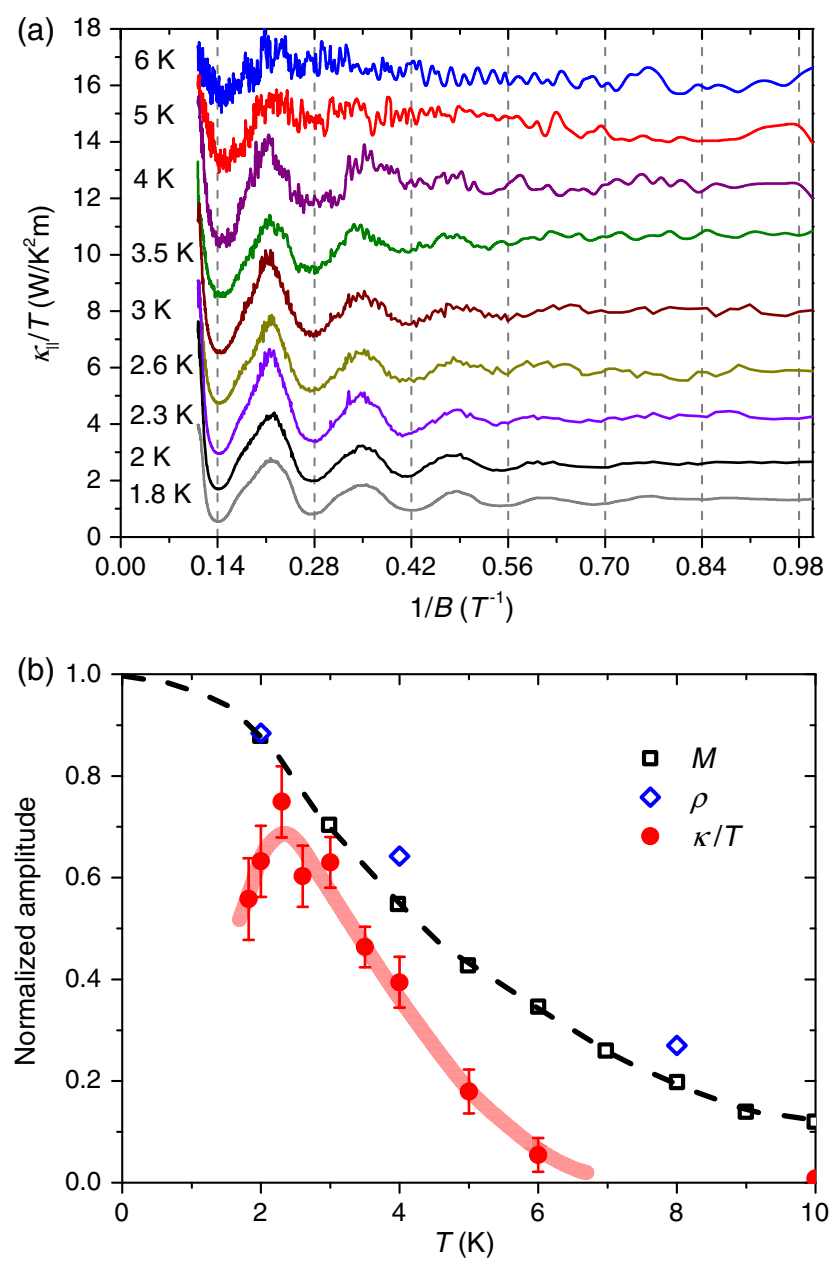

FIG. 3. Evolution of the $\kappa_{\|}(B)$ MQOs as a function of the temperature. (a) Isothermal $\kappa_{\|} / T$ curves as a function of $B^{-1}$ measured at selected temperatures between $T=1.8$ and $6 \mathrm{~K}$. A characteristic frequency $F \approx 7 \mathrm{~T}$ can be readily observed from the $T$-independent oscillation period of approximately $0.14 \mathrm{~T}^{-1}$ for all the curves. The corresponding FFT analysis is shown in Fig. S7. For the sake of clarity, the $\boldsymbol{\kappa}_{\|} / T$ curves are shifted upward by 7 (6 K), 6 (5 K), 5 (4 K), 5.5 (3.5 K), 4.5 (3 K), 3.5 $(2.6 \mathrm{~K}), 2(2.3 \mathrm{~K})$, and $1(2 \mathrm{~K})$ units of $\mathrm{W} / \mathrm{K}^{2} \mathrm{~m}$, respectively. (b) Temperature dependence of the normalized FFT amplitude of the characteristic MQOs with $F \approx 7 \mathrm{~T}$, compared to the ones observed in magnetization (extracted from Ref. [22]) and electrical conductivity [cf. Fig. 2(a)].

$\sigma_{\|}(B)$. The largest change in the last MQO period of $\kappa_{\|}(B)$ [Fig. 2(b)] amounts to $d \kappa=12 \mathrm{~W} / \mathrm{K} \mathrm{m}$, i.e., 3.4 times the value of $\kappa_{\|}(B=0)$. The MQOs with $F \approx 7 \mathrm{~T}$ are practically the only visible oscillating component in $\kappa_{\|}(B)$; cf. $\kappa_{\|}(B) / T$ vs $B^{-1}$ shown in Fig. 3(a) and the corresponding FFT analysis in Fig. S7 [24], in striking contrast to $\sigma_{\|}(B)$ [Fig. 2(a)] and $S_{\|}(B)$ (Fig. S3 [24]). The excellent agreement of this frequency with that of the Weyl pocket probed by other techniques $[19,21,22]$ clearly indicates that the MQOs observed in $\kappa_{\|}(B)$ are a consequence of the Landau-quantized electronic density of states (DOS) of the 
Weyl fermions in the bulk of TaAs. MQOs of the same frequency, with 5-6 times smaller amplitude, are also observed in the perpendicular field configuration, i.e., $\kappa_{\perp}(B)$ of sample TAa measured with $d T \| a$ and $B \| c$; see Fig. S5 [24]. Nevertheless, these oscillations are still quite large, when compared to $\kappa_{e, \mathrm{WF}}(B)$ estimated from the WF law; cf. Fig. S6 [24]. The recently observed MQOs in $\kappa_{\perp}(B)$ of $\mathrm{NbP}\left[\kappa_{\|}(B)\right.$ is not measured] reveal a somewhat smaller but still comparable oscillation amplitude $\left(d \kappa / \kappa_{0 \mathrm{~T}} \approx 30 \%\right)$ at $T=2 \mathrm{~K}$ [18]. Note that for this compound the FFT spectrum is much more complicated than for TaAs.

\section{ANALYSIS OF OSCILLATORY HEAT CONDUCTION}

The common description of the thermal conductivity in nonmagnetic conductors considers diffusion contributions from both electrons and phonons, i.e., $\kappa_{e}$ and $\kappa_{\mathrm{ph}}$. For semimetals and semiconductors, a bipolar conduction term $\kappa_{\mathrm{bi}}$ has to be taken into account, too. This term is due to thermally excited electron-hole pairs which diffuse to the cold end, where they recombine, releasing their excitation energies [26]. As already mentioned in the introduction, $\kappa_{e}$ can be readily estimated from $\sigma$ via the WF law [Eq. (1)] for a wide variety of conducting materials, under dominating elastic scattering [5]. This estimation is often realized at low enough temperatures with dominating impurity scattering. On the other hand, the lattice contribution $\kappa_{\mathrm{ph}}$ can be approached from first-principle calculations [27], as long as the intrinsic phonon-phonon umklapp scattering is the dominating phonon relaxation process. It may also interact with the electron-diffusion term through $e-p$ coupling, as is argued for the Landau-quantized 2DES [17]. A bipolar contribution $\kappa_{\mathrm{bi}}$ will become significant only near a chargeneutrality condition, as realized, e.g., in graphene [28].

To identify the potential origin of the giant $\kappa(B)$ MQOs in TaAs, we first examine the possibility of a large electronic contribution $\kappa_{e}(B)$ that is well enhanced over its WF counterpart $\kappa_{e-\mathrm{WF}}(B)$, implying $L(B) \gg L_{0}$. In order for this scenario to work, $L(B)$ has to exceed $L_{0}$ by a factor of approximately 100; cf. Fig. 2(b) (note the logarithmic $y$ axis). A similar conclusion can be drawn from measurements with the perpendicular field configuration (Fig. S6 [24]). This situation is different from the chiral-anomaly-induced negative MR that appears only in parallel fields. In fact, for nonmagnetic conductors, the application of a magnetic field hardly affects the Lorenz number $L$, because the charge-relaxation mechanisms involved remain unchanged. In $\mathrm{Cu}$, for example, $L$ changes at $4.8 \mathrm{~K}$ by only $10 \%$ when $B$ is increased up to $5 \mathrm{~T}$ [29]. Recently, electrical and thermal signatures of a hydrodynamic electron fluid with a violated WF law have been identified in the topological semimetals like $\mathrm{WP}_{2}$ [10]. There, however, the Lorenz number $L$ is found to be largely reduced from $L_{0}$, contrary to our observations made in TaAs.

There is compelling evidence against the electrondiffusion scenario described above based on the following two observations. The first one concerns the $T$ dependence of the oscillations as shown in Figs. 3(a) and 3(b); see also the corresponding FFT spectrum shown in Fig. S7 [24]. While the oscillation amplitude of both the magnetization $M(B)$ and $\sigma(B)$ follows the Lifshitz-Kosevich function for thermally damped Landau quantization [22], i.e., steadily decreases upon warming, that of $\kappa_{\|}(B) / T$ does not; cf. Fig. 3(b). The latter becomes largest at $T \approx 2.3 \mathrm{~K}$ and diminishes upon further cooling, suggesting a nonelectronic, most likely bosonic contribution. The second observation is that of an oscillation phase of the observed $\kappa_{\|}(B)$ MQOs which is opposite to that of $\sigma_{\|}(B)$ or $\kappa_{e, \mathrm{WF}}(B)$. For example, at $B=7.3 \mathrm{~T}$ (dashed line in Fig. 2), where $\sigma_{\|}(B)$ shows a maximum because of the intersection of a Landau level with the Fermi level (causing a maximum in the DOS $), \kappa_{\|}(B)$ reveals a minimum [Fig. 2(b)]. This result implies that in this very field where TaAs is electrically most conductive it is thermally almost insulating, contrary to what is expected in the case of conduction by electron diffusion.

An alternative explanation for the giant MQOs in $\kappa(B)$ may involve the bipolar-diffusion term $\kappa_{\mathrm{bi}}(B)$, as argued in the case of $\mathrm{NbP}$ [18]. This effect has been intensively investigated for graphene, which fulfills all the requirements for bipolar transport, e.g., zero band gap and charge neutrality [28]. There, under proper electrical gating, bipolar diffusion can enhance $L$ by a factor of $2-4$ at room temperature. This factor diminishes with lowering $T$ due to the freezing out of thermal excitations. Experimentally, the enhancement of $L(T)$ is estimated to be only $35 \%$ in the sub-Kelvin region [30]. Related to the bipolar excitations in graphene, as already mentioned in the introduction, a further enhancement of $L$ up to $22 L_{0}$ was recently observed at elevated temperatures, i.e., between 50 and $80 \mathrm{~K}$ [11]. There, the charge-neutral electron-hole plasma obeying hydrodynamics is argued to be realized. In TaAs, the dominating Weyl node is located $2 \mathrm{meV}$ above the Fermi level which intersects with several other topological trivial and nontrivial pockets. This result implies that TaAs does not fulfill the charge-neutrality requirement for significant transport by electron-hole excitons. While a dominating bipolar heat transport should rapidly diminish upon an increasing magnetic field [26], because the electron-hole excitons are easily destroyed, the giant $\kappa(B)$ MQOs observed in TaAs indeed persist up to $9 \mathrm{~T}$; see Fig. 2(b). This observation yields further evidence against the bipolar-diffusion scenario.

Having discarded both the electronic and bipolar contributions to heat transport as potential origins of the giant MQOs observed in $\kappa(B)$, we now turn to the possible influence of the phonon contribution $\kappa_{\mathrm{ph}}(B)$ for which $e-p$ coupling may cause an oscillating behavior due to the 
field-induced Landau quantization of the electronic DOS at the Fermi energy. This scenario is suggested to explain the relatively small $(2 \%)$ MQOs of $\kappa(B)$ reported for GaAs/AlGaAs heterostructures [17]. In this case, the phonon mean-free path is modulated in a magnetic field, via $e-p$ coupling, by the Landau-quantized 2D DOS which is confined to the interface. This effect should be much weaker in a 3D system with multiple Fermi pockets like TaAs, where discrete Landau levels are absent. To further explore this possibility, we perform ultrasound measurements on TaAs. In the configuration with the phonon propagation and magnetic field being parallel along the $c$ axis, the MQOs of the phonon velocity $v(B)$, while also having a dominating frequency of $7 \mathrm{~T}$, are only of the order of $1 / 10^{4}$; see Fig. 4, left axis. This result is consistent with our first-principles calculations of the phonon-dispersion relations of TaAs which reveal negligible field dependence; see Fig. S8 [24]. Moreover, up to $B=9 \mathrm{~T}$, the observed MQOs of the ultrasound echo amplitude, which are a measure of the phonon mean-free path, are only within 5\%; see Fig. 4, right axis. Here, we adopt a transverse phonon of relatively low frequency $(f=19 \mathrm{MHz})$; similar results are reported for this

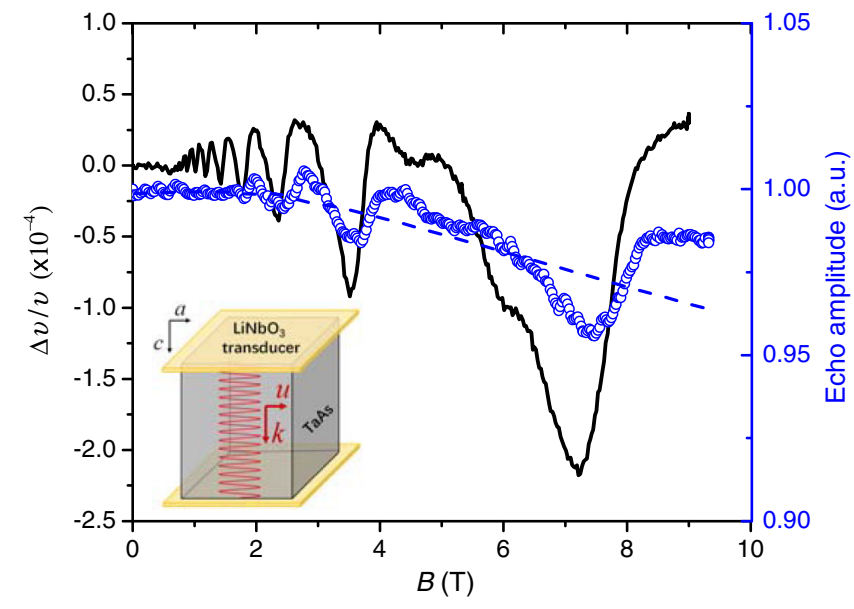

FIG. 4. Ultrasound velocity (left) and the corresponding ultrasound echo amplitude (right) measured as a function of the field at $T=2 \mathrm{~K}$. A transverse sound of $19 \mathrm{MHz}$ is used, with the propagation $(k)$ and polarization $(u)$ along the $c$ and $a$ axes, respectively (see the inset). The detected sound velocity is approximately $2010 \mathrm{~m} / \mathrm{s}$, as expected for the transverse acoustic phonons of TaAs [32]. These measurements are performed under the condition that the ultrasound propagation and magnetic field are parallel and along the $c$ axis, corresponding to the field configuration for $\kappa_{\|}(B)$ measurements. The dominating oscillations in both quantities have a frequency of $7 \mathrm{~T}$, almost in phase with $\kappa_{\|}(B)$ MQOs; see Fig. 2(b). The quantum oscillations of the sound velocity are of the order of $1 / 10^{4}$ up to $9 \mathrm{~T}$, whereas the corresponding echo amplitude oscillates within $5 \%$ of its zero field value. The dashed blue line indicates the decreasing background of the ultrasound echo amplitude as a function of $B$, which implies a decreasing phonon mean-free path. material by using longitudinal phonons of much higher frequencies (>300 MHz) [31].

Based on our ultrasound measurement results, employing the kinetic formula for the heat conductivity carried by acoustic phonons, $\kappa_{\mathrm{ph}}=1 / 3 C_{\mathrm{ph}} v_{p h} l_{\mathrm{ph}}$, the magnetothermal conductivity $\kappa_{\|}(B)$ can be estimated to oscillate within $5 \%$ of its zero-field value, assuming $\kappa_{\mathrm{ph}}(B)$ dominates $\kappa_{\|}(B)$. Here, $C_{\mathrm{ph}}$ is the phonon contribution to the specific heat, $v_{\mathrm{ph}}$ the phonon velocity, and $l_{\mathrm{ph}}$ the phonon mean-free path. While this estimate may indeed account for the $\kappa(B)$ MQOs observed in bismuth [16], it is much too small to explain our observations on TaAs. Moreover, the smooth background of the ultrasound echo amplitude tends to decrease as a function of $B$ (see the dashed blue line in Fig. 4). A similar tendency can also be derived from the ultrasound attenuation at $B<10 \mathrm{~T}$ as reported in Ref. [31]. These are in stark contrast to our observation that the smooth background $\kappa_{b g}(B)$ of $\kappa_{\|}(B)$ increases with the field (Fig. 5, discussed below), although both phonon propagation and heat transport are measured with the same parallel field along the $c$ axis. In conclusion, though the propagating acoustic phonons can indeed be affected by the Landauquantized electronic DOS and give rise to MQOs of both

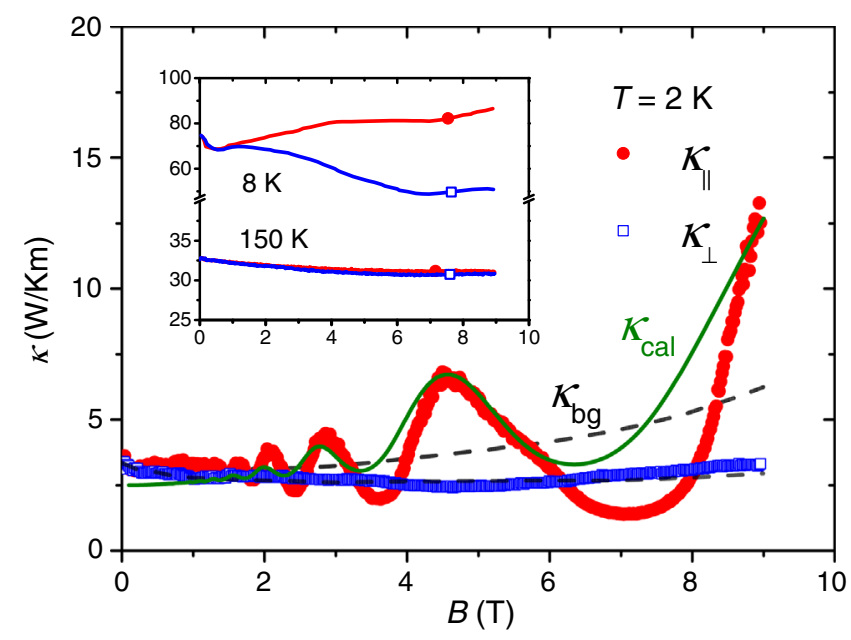

FIG. 5. Comparison of $\kappa(B)$ measured for sample TAc in parallel $(d T\|c, B\| c)$ and perpendicular $(d T\|c, B\| a)$ field configurations. The dashed black lines denote a smooth background $\kappa_{\mathrm{bg}}(B)$ for $\kappa_{\|}(B)$ and $\kappa_{\perp}(B)$. It is clearly seen that $\kappa_{\mathrm{bg}}(B)$ for the parallel field configuration gradually increases, whereas that for the perpendicular field slightly decreases with $B$. These apparently different trends of $\kappa_{\mathrm{bg}}(B)$ between the two field configurations provide strong support for CZS being involved in the magnetothermal conductivity. Furthermore, a semiquantitative fitting based on the CZS scenario is also shown [solid green $\kappa_{\text {cal }}(B)$ line], which agrees reasonably well with the experimental results. Inset: The trend of the background contribution for the two different field configurations is much better demonstrated at $T=8 \mathrm{~K}$, where the $F \approx 7 \mathrm{~T}$ oscillations have almost disappeared; cf. Fig. 3(b). At a much higher temperature $(T=150 \mathrm{~K})$, the data for $\kappa_{\|}(B)$ and $\kappa_{\perp}(B)$ fall on top of each other. 
sound velocity and echo amplitude, they are unapt to account for the giant, experimentally observed MQOs of the thermal conductivity of TaAs.

Recently, a distinct magnetothermal conductivity arising from the Fermi arcs at the surfaces of Weyl semimetals, mediated by a Weyl node, has been proposed [33]. However, this proposal also cannot explain our observations, because the giant $\kappa(B)$ MQOs apparently probe the Weyl fermions in the bulk of TaAs.

To further explore the underlying physics of the giant $\kappa(B)$ MQOs, we now compare $\kappa_{\|}(B, B \| c)$ and $\kappa_{\perp}(B, B \| a)$ measured on the same sample TAc; see Fig. 5. Except for the giant MQOs observed only in $\kappa_{\|}(B)$, one recognizes that the smooth background of $\kappa_{\|}(B)$ is strongly enhanced by the field, as shown by a dashed line (black) denoted as $\kappa_{b g}(B)$. By contrast, $\kappa_{\perp}(B)$ changes only weakly with the field, with hardly detected MQOs and a field-induced, weakly decreasing $\kappa_{\mathrm{bg}}(B)$. The field-induced decrease of $\kappa_{\mathrm{bg}}(B)$ observed in the perpendicular field tracks the common field dependence of both $\kappa_{e}(B)$ or $\kappa_{\mathrm{bi}}(B)$. Recently, $\kappa_{\mathrm{ph}}(B)$ has also been demonstrated to decrease with the field [34], due to a fieldinduced local diamagnetism that exerts an enhanced anharmonic magnetic force on the lattice vibrations. The deceasing ultrasound echo amplitude shown Fig. 4, right axis, indicates that this scenario may apply to TaAs, too. Conversely, the smooth increase of $\kappa_{\mathrm{bg}}(B)$ observed for the parallel field configuration (Fig. 5) supports the notion of exotic heat carriers that tend to carry an increasing amount of heat with an increasing magnetic field, strongly arguing against a phonon origin.

\section{CHIRAL ZERO SOUND}

Below, we propose that the CZS of Weyl fermions is a strong candidate to explain the exotic behavior of $\kappa(B)$ that is observed in TaAs [23]. Different to the sound in a solid due to lattice vibrations, CZS in a Weyl semimetal is a unique acoustic wave formed by the antisymmetric combination of the oscillating currents from different Weyl valleys caused by the chiral magnetic effect. As discussed in Ref. [23], the CZS is a collective bosonic excitation of Weyl fermions and propagates along the magnetic field, without any net charge current involved. Its velocity is antiproportional to the electronic DOS at the Fermi level, leading to strong quantum oscillations for the thermal conductivity along the magnetic field. For a perpendicular field configuration, the CZS is expected to affect the thermal conductivity to some extent, too, due to its contribution to the specific heat [23]. Furthermore, the nonoscillatory part of the thermal conductivity due to CZS along the field increases linearly with field $B$, as the sound velocity is proportional to $B$. These distinct features of the CZS appear to be well consistent with the main experimental results reported in this work, i.e., the giant and much smaller MQOs in $\kappa_{\|}(B)$ and $\kappa_{\perp}(B)$, respectively, as well as the increasing background $\kappa_{\mathrm{bg}}(B)$ in the former.

In Fig. 5, we also present a semiquantitative fitting to our experimental results based on the CZS scenario. There are three relevant material parameters in the CZS theory [23]: the Fermi wave vector $k_{F}$, the quasiparticle lifetime $\tau_{0}$, and the intervalley relaxation time $\tau_{v}$. The former two parameters can be determined experimentally from the frequency of quantum oscillations and the dc electric conductivity, respectively. By choosing a proper intervalley relaxation time according to the relative amplitude of the quantum oscillations in thermal conductivity, we can calculate $\kappa_{\|}(B)$ by using Eq. (11) in Supplemental Material [24]. As shown in Fig. 5, the calculated results (green solid line) can reproduce the experimental values reasonably well; see Supplemental Material for a detailed note on the calculation [24]. Furthermore, we indeed find $\tau_{v} \gg \tau_{0}$, which suggests that the intravalley scattering is much stronger than the intervalley scattering and, therefore, the CZS is truly stabilized by the so-called chiral limit [23,24].

\section{CONCLUDING REMARKS}

As the strength of the $e-p$ interaction is proportional to (or, in other words, the resulted phonon relaxation time is antiproportional to) the quantized DOS, one expects the MQOs of the common phonon contribution $\kappa_{\mathrm{ph}}(B)$ to be in phase with and additive to those of the CZS. This contribution may partly explain the discrepancy of the semiquantitative fitting shown in Fig. 5. Obviously, our ultrasound measurements detect an acoustic phonon, as the observed phonon velocity $v_{\mathrm{ph}} \approx 2010 \mathrm{~m} / \mathrm{s}$ is a typical value for transverse acoustic phonons in TaAs [32]. In our ultrasound spectra, we could not directly detect the CZS which is expected to occur at high frequencies (a rough estimate based on the dominating intervalley relaxation time $\tau_{v}$ indicates a frequency of at least several hundred megahertz). Another aspect making ultrasound detection of CZS difficult concerns the sound velocity of CZS, which is estimated to be $5 \times 10^{4} \mathrm{~m} / \mathrm{s}$ for typical Weyl semimetals [23], one order of magnitude larger than that of acoustic phonons in common solids. Therefore, the detection of the CZS mode by an ultrasound technique requires a much higher time resolution; efforts in this direction are still underway.

In conclusion, we observe giant magnetic quantum oscillations in the magnetothermal conductivity of TaAs. All the conventional mechanisms due to diffusion of electrons, phonons, and bipolar excitations are unable to account for these phenomena. Alternatively, we identify that chiral zero sound associated with the Weyl fermions is a likely source for these observations. This source is a collective bosonic excitation of Weyl fermions which acts as an efficient heat carrier and results in a huge ratio of heat over charge conduction in a finite temperature regime 
around a few Kelvins. It is worth mentioning that, except for the fundamental significance, the large thermal conductance observed in this work may also find feasible applications in heat management of electronic devices as, e.g., a controllable heat switch.

\section{METHODS}

The thermal conductivity is measured utilizing a homedesigned sample holder with a chip resistor of $2000 \Omega$ as heater and a thin $(\phi=25 \mu \mathrm{m})$ chromel-AuFe ${ }_{0.07 \%}$ thermocouple for detecting the temperature difference $d T$ across the sample; see Fig. 1(a). Silver epoxy H20E (EPO-TEK) is used to make good thermal contact between the sample and the cold plate of the sample holder. The connection points of the thermocouple wires, after being wrapped by a tiny drop of Stycast 2850 (Emerson \& Cuming), are pressed onto the sample surface and further fixed by GE varnish in order to ensure thermal connection but electrical insulation to the sample. Thin nylon wires are used to support the thermocouple and heater. Electrical insulation between the sample and the thermocouple is confirmed before thermal transport measurements. In order to minimize heat loss, thin manganin wires $(\phi=25 \mu \mathrm{m})$ are used as lead wires for the heater and thermocouple; the whole measurement unit is kept in a high vacuum. Furthermore, prior to our measurements, the thermocouple is carefully calibrated in magnetic fields by using two Cernox CX1050 thermometers; a similar field dependence as previously reported [35] is observed; cf. Fig. S1 [24].

In order to double check our observations of the giant $\kappa(B)$ MQOs, we also employ the thermal transport option equipped on the Physical Properties Measurement System (PPMS, Quantum Design) to measure the magnetothermal conductivity. This method employs two field-calibrated Cernox thermometers to detect $d T$; see Fig. S2(a), inset [24]. As shown in Fig. S2(a), the thermal conductivity $\kappa_{\|}(B)$ obtained by the two different methods agrees reasonably well. We note, however, that the thermal measurements by two thermometers are extremely time consuming due to the long relaxation time for each measurement point. By contrast, employment of a thin thermocouple ensures a rapid thermal relaxation during a scan of the magnetic field, which is of uttermost importance for reliable detection of the $\kappa(B)$ MQOs. The reliability of the thermal transport measurements can be confirmed from the recorded $d T(B)$ profile at $T=2 \mathrm{~K}$ [cf. Fig. 1(b)], which is almost field symmetric with reproducible MQOs for both positive and negative fields. Thermal conductivity $\kappa$ is obtained via $\kappa=A Q / d T$, where $A$ is the sample geometrical factor and $Q$ the heating power. The measured $d T$ in the whole field range is stable within $5 \%$.

We also perform ultrasound measurements by using a phase-comparison technique [36] to examine the quantum oscillations of (the velocity and amplitude of) propagating acoustic phonons. $\mathrm{LiNbO}_{3}$ piezoelectric plates with a fundamental resonance frequency of $19 \mathrm{MHz}$ are used as ultrasonic transducers to generate and detect the ultrasound signal; see Fig. 4, inset. The relative change of the sound velocity in the sample can be monitored by the frequency change of the propagating phonons; the corresponding amplitude of the transmitted phonons is directly measured as a voltage generated by ultrasound echoes.

\section{ACKNOWLEDGMENTS}

We acknowledge fruitful discussions with G. Li, Y. Li, K. Behnia, H. Weng, and Z. Fang and technical assistance in ultrasound measurements from M. Yoshizawa. This work was supported by the National Science Foundation of China (Grants No. 11474332, No. 11474015, No. 11774018, and No. 61227902), the MOST of China (Grants No. 2015CB921303 and No. 2017YFA0303103), and the Chinese Academy of Sciences through the strategic priority research program (XDB07020200).

Note added.- Recently, we learned about new experimental results of the magnetothermal conductivity on $\mathrm{TaAs}_{2}$ and $\mathrm{NbAs}_{2}$ [37]. At sub-Kelvin temperatures, these two candidates for magnetic-field-induced Weyl semimetals exhibit huge magnetic quantum oscillations of the thermal conductivity, which amount to 2 orders of magnitude of and are nearly antiphase to what is estimated based upon the WF law. The physics underlying these observations remains unsolved but may be explained by chiral zero sound, too.

[1] H. B. Nielsen and M. Ninomiya, The Adler-Bell-Jackiw Anomaly and Weyl Fermions in a Crystal, Phys. Lett. B 130, 389 (1983).

[2] P. Hosur and X. L. Qi, Recent Developments in Transport Phenomena in Weyl Semimetals, C.R. Phys. 14, 857 (2013).

[3] A. A. Burkov, Chiral Anomaly and Transport in Weyl Metals, J. Phys. Condens. Matter 27, 113201 (2015).

[4] S. Wang, B. C. Lin, A. Q. Wang, D. P. Yu, and Z. M. Liao, Quantum Transport in Dirac and Weyl Semimetals: A Review, Adv. Phys. X 2, 518 (2017).

[5] N. W. Ashcroft and N. D. Mermin, Solid State Physics, 1st ed. (Brooks-Cole, Belmont, MA, 1976).

[6] K. S. Kim, Role of Axion Electrodynamics in a Weyl Metal: Violation of Wiedemann-Franz Law, Phys. Rev. B 90, 121108(R) (2014).

[7] G. Sharma, P. Goswami, and S. Tewari, Nernst and Magnetothermal Conductivity in a Lattice Model of Weyl Fermions, Phys. Rev. B 93, 035116 (2016).

[8] R. Lundgren, P. Laurell, and G. A. Fiete, Thermoelectric Properties of Weyl and Dirac Semimetals, Phys. Rev. B 90, 165115 (2014).

[9] Z. Luo, J. Tian, S. Huang, M. Srinivasan, J. Maassen, Y. P. Chen, and $\mathrm{X}$. Xu, Large Enhancement of Thermal Conductivity and Lorenz Number in Topological Insulator Thin Films, ACS Nano 12, 1120 (2018). 
[10] J. Gooth, F. Menges, N. Kumar, V. Sü $\beta$, C. Shekhar, Y. Sun, U. Drechsler, R. Zierold, C. Felser, and B. Gotsmann, Thermal and Electrical Signatures of a Hydrodynamic Electron Fluid in Tungsten Diphosphide, Nat. Commun. 9, 4093 (2018).

[11] J. Crossno, J. K. Shi, K. Wang, X. Liu, A. Harzheim, A. Lucas, S. Sachdev, P. Kim, T. Taniguchi, K. Watanabe, T. A. Ohki, and K. C. Fong, Observation of the Dirac Fluid and the Breakdown of the Wiedemann-Franz Law in Graphene, Science 351, 1058 (2016).

[12] G. J. Checkelsky and N. P. Ong, Thermopower and Nernst Effect in Graphene in a Magnetic Feld, Phys. Rev. B 80, 081413(R) (2009).

[13] T. Liang, Q. Gibson, J. Xiong, M. Hirschberger, P. Koduvayur, R. J. Cava, and N. P. Ong, Evidence for Massive Bulk Dirac Fermions in Pb1-xSnxSe from Nernst and Thermopower Experiments, Nat. Commun. 4, 2696 (2013).

[14] Z. Zhu, X. Lin, J. Liu, B. Fauqué, Q. Tao, C. Yang, Y. Shi, and K. Behnia, Quantum Oscillations, Thermoelectric Coefficients, and the Fermi Surface of Semimetallic WTe2, Phys. Rev. Lett. 114, 176601 (2015).

[15] M. Matusiak, J. R. Cooper, and D. Kaczorowski, Thermoelectric Quantum Oscillations in ZrSiS, Nat. Commun. 8, 15219 (2017).

[16] S. M. Bhagat and D. D. Manchon, Jr, Heat Transport in Bismuth at Liquid-Helium Temperatures, Phys. Rev. 164, 966 (1967).

[17] J. P. Eisenstein, A. C. Gossard, and V. Narayanamurti, Quantum Oscillations in the Thermal Conductance of GaAs/ AlGaAs Heterostructures, Phys. Rev. Lett. 59, 1341 (1987).

[18] U. Stockert, R. D. dos Reis, M. O. Ajeesh, S. J. Watzman, M. Schmidt, C. Shekhar, J. P. Heremans, C. Felser, M. Baenitz, and M. Nicklas, Thermopower and Thermal Conductivity in the Weyl Semimetal NbP, J. Phys. Condens. Matter 29, 325701 (2017).

[19] X. Huang, L. Zhao, Y. Long, P. Wang, D. Chen, Z. Yang, H. Liang, M. Xue, H. Weng, Z. Fang, X. Dai, and G. Chen, Observation of the Chiral-Anomaly-Induced Negative Magnetoresistance in $3 D$ Weyl Semimetal TaAs, Phys. Rev. X 5 , 031023 (2015).

[20] H. Weng, C. Fang, Z. Fang, B. A. Bernevig, and X. Dai, Weyl Semimetal Phase in Noncentrosymmetric TransitionMetal Monophosphides, Phys. Rev. X 5, 011029 (2015).

[21] C. L. Zhang et al., Signatures of the Adler-Bell-Jackiw Chiral Anomaly in a Weyl Fermion Semimetal, Nat. Commun. 7, 10735 (2016).

[22] F. Arnold, M. Naumann, S.-C. Wu, Y. Sun, M Schmidt, H. Borrmann, C. Felser, B. Yan, and E. Hassinger, Chiral Weyl Pockets and Fermi Surface Topology of the Weyl Semimetal TaAs, Phys. Rev. Lett. 117, 146401 (2016).

[23] Z. Song and X. Dai, Hear the Sound of Weyl Fermions, Phys. Rev. X 9, 021053 (2019).
[24] See Supplemental Material at http://link.aps.org/ supplemental/10.1103/PhysRevX.9.031036 for detailed information on the field calibration of the thermocouple, cross-checking of the magnetothermal conductivity by different thermometries and the FFT analysis, and additional experimental results for another sample, as well as the quantitative analysis of the chiral zero sound contribution.

[25] J. Xiang, S. Hu, M. Lv, J. Zhang, H. Zhao, G. Chen, W. Li, Z. Chen, and P. Sun, Anisotropic Thermal and Electrical Transport of Weyl Semimetal TaAs, J. Phys. Condens. Matter 29, 485501 (2017).

[26] J. R. Drabble and H. J. Goldsmid, Thermal Conduction in Semiconductors (Pergamon, New York, 1961), p. 115.

[27] T. Ouyang, H. Xiao, C. Tang, M. Hu, and J. Zhong, Anisotropic Thermal Transport in Weyl Semimetal TaAs, a First Principles Calculation, Phys. Chem. Chem. Phys. 18, 16709 (2016).

[28] H. Yoshino and K. Murata, Significant Enhancement of Electronic Thermal Conductivity of Two-Dimensional Zero-Gap Systems by Bipolar-Diffusion Effect, J. Phys. Soc. Jpn. 84, 024601 (2015).

[29] A. Fevrier and D. Morize, The Effect of Magnetic Field on the Thermal Conductivity and Electrical Resistivity of Different Materials, Cryogenics 13, 603 (1973).

[30] K. C. Fong, E. E. Wollman, H. Ravi, W. Chen, A. A. Clerk, M. D. Shaw, H. G. Leduc, and K. C. Schwab, Measurement of the Electronic Thermal Conductance Channels and Heat Capacity of Graphene at Low Temperature, Phys. Rev. X 3, 041008 (2013).

[31] B. J. Ramshaw et al., Quantum Limit Transport and Destruction of the Weyl Nodes in TaAs, Nat. Commun. 9, 2217 (2018).

[32] B. Peng, H. Zhang, H. Shao, H. Lu, D. W. Zhang, and H. Zhu, High Thermoelectric Performance of Weyl Semimetal TaAs, Nano Energy 30, 225 (2016).

[33] T. M. McCormick, S. J. Watzman, J. P. Heremans, and N. Trivedi, Fermi Arc Mediated Entropy Transport in Topological Semimetals, Phys. Rev. B 97, 195152 (2018).

[34] H. Jin, O. D. Restrepo, N. Antolin, S. R. Boona, W. Windl, R. C. Myers, and J.P. Heremans, Phonon-Induced Diamagnetic Force and Its Effect on the Lattice Thermal Conductivity, Nat. Mater. 14, 601 (2015).

[35] U. Stockert and N. Oeschler, Thermopower of Chromel-AuFe0.07\% Thermocouples in MagneticFfields, Cryogenics 51, 154 (2011).

[36] P. Sun, Y. Nakanishi, M. Nakamura, M. Yoshizawa, M. Ohashi, G. Oomi, C. Sekine, and I. Shirotani, Anomalous Elastic Softening of $\mathrm{SmRu}_{4} \mathrm{P}_{12}$ under High Pressure, Phys. Rev. B 75, 054114 (2007).

[37] X. Rao et al., Quantum Oscillation of Thermal Conductivity and Violation of Wiedemann-Franz, Law in $\mathrm{TaAs}_{2}$ and $\mathrm{NbAs}_{2}$, arXiv:1906.03961. 